\title{
Elastoplasticité anisotrope non normale en grandes déformations avec endommagement
}

\section{Application à la mise en forme des tôles minces}

\author{
Houssem Badreddine*,** — Khémaïs Saanouni* \\ Abdelwaheb Dogui** — M. Amen Gahbich** \\ * ICD/SMIS, FRE, CNRS N 2848 - Université de Technologie de Troyes \\ 12, rue marie curie BP 2060, F-10000 Troyes \\ ** LGM/GM-MA05 - Ecole Nationale d'Ingénieurs de Monastir \\ Av. Ibn El Jazzar, 5019 Monastir - Tunisie
}

\begin{abstract}
RÉSUMÉ. Ce travail est dévolu à l'intégration numérique d'un modèle de comportement élastoplastique endommageable formulé en transformations finies. Ce modèle à écrouissage mixte (cinématique et isotrope) non linéaire est basé sur une théorie de plasticité non associée et non normale avec un couplage fort comportement-endommagement. La procédure d'intégration adoptée est basée sur la méthode de prédiction élastique-correction plastique. Le modèle ainsi que son algorithme d'intégration sont implémentés via la routine VUMAT dans ABAQUS. La résolution globale est assurée par un schéma dynamique explicite (DE) disponible dans ABAQUS. L'application est faite à la prédiction de la rupture de tôles minces anisotropes soumises à un gonflement hydraulique avec matrices circulaire et elliptique.

ABSTRACT. This work is devoted to the numerical integration of an elastoplastic model strongly coupled with ductile damage and formulated in finite deformation. This model with mixed nonlinear work hardening (kinematic and isotropic) is based on a non associative and non normal plasticity theory. The integration is based on the elastic prediction - plastic correction procedure. The proposed constitutive models as well as the associated integration scheme are implemented into ABAQUS/Explicit thanks to VUMAT subroutine. The global resolution is done using the classical Dynamic Explicit (DE) strategy with ABAQUS/Explicit F.E. code. Application is made to the prediction of rupture in anisotropic thin sheet subjected to a hydro bulging test with circular and elliptic matrices. A conparison between the numerical and experimental results shows the capacity of such a modeling to reproduce the anisotropic thin sheet rupture.
\end{abstract}

MOTS-CLÉS: grandes déformations, élastoplasticité, anisotropie, non-normalité, endommagement ductile, calcul des contraintes, retour normal, éléments finis, simulation numérique, mise en forme. KEYWORDS: finite deformation, elastoplasticity, anisotropy, non normality, ductile damage, stress computation, normal mapping, finite elements, numerical simulation, metal forming.

DOI:10.3166/REMN.16.913-940 @ 2007 Lavoisier, Paris 
914 REMN - 16/2007. Modélisations numériques en mécanique

\section{Introduction}

La simulation numérique est devenue un atout important pour la réussite des industries de la mise en forme par grandes déformations plastiques. Cette pratique, qui se développe de plus en plus, nécessite la mise en œuvre de modèles de comportements évolués afin de rendre compte au mieux des phénomènes mis en jeux. Ces modèles, vu les matériaux utilisés et les chargements qu'ils subissent, doivent êtres formulés en transformations finies. L'utilisation de tels modèles dans des codes de calcul requiert leur développement sous une forme incrémentale en adoptant des procédures d'intégrations robustes assurant leur stabilité et leur objectivité incrémentale.

La plupart des codes de calcul par éléments finis utilisés en mise en forme reposent sur la formulation variationnelle du problème d'équilibre en déplacement ou en vitesse. La discrétisation spatiotemporelle de cette dernière conduit à un système discret dont l'inconnue principale est le vecteur des déplacements nodaux. Des méthodes implicites et explicites sont détaillées dans la littérature pour la résolution de ce type de système (Bathe, 1981; Bonnet et al., 1997; Simo et al., 1998 ; Belytschko et al., 2000...). Néanmoins, les méthodes explicites sont préférées pour la simulation numérique des procédés de mise en forme, parce qu'elles ne nécessitent pas la détermination de matrices tangentes comme pour le cas des méthodes implicites. En revanche, pour l'une ou l'autre des deux méthodes il est primordial d'effectuer une intégration locale des équations constitutives du modèle de comportement en chaque point de Gauss afin de calculer la contrainte en fin de chaque incrément.

Dans la littérature de nombreux travaux ont traité de l'intégration numérique de modèles de plasticité divers et variés (Hughes et al., 1980 ; Lee et al., 1985 ; Hartley et al., 1989 ; Freed et al., 1992 ; Zhu et al., 1992 ; Khan et al., 1995 ; Chaboche et al., 1996 ; Simo et al., 1998 ; Besson et al., 2001 ; Saanouni et al., 2003...). En particulier les modèles à variables internes ont fait l'objet de travaux basés sur la méthode de prédiction élastique - correction plastique (Zhu et al., 1992 ; Chaboche et al., 1996; Simo et al., 1998 ; Besson et al., 2001 ; Saanouni et al., 2003...). Quelques travaux ont été consacrés à l'intégration de modèles à variables internes de (visco) plasticité couplée à l'endommagement en petites perturbations ou en grandes déformations (Benallal et al., 1988 ; Doghri, 1993 ; Zhu et al., 1992 ; Saanouni et al., 1994 ; Hammi, 2000 ; Saanouni et al., 2003 ; Khelifa et al., 2005 ; Badreddine, 2006). En grandes déformations la quasi-majorité des travaux dédiés à l'intégration numérique de modèles de plasticité concernent des modèles à écrouissage isotrope. Plus rares sont les travaux dédiés à l'intégration de modèles anisotropes en grandes déformations avec écrouissage mixte (isotrope et cinématique) et à couplage fort comportement - endommagement (Khelifa et al., 2005 ; Belamri et al., 2005 ; Khelifa et al., 2006). Ces travaux qui respectent la règle de normalité ont montré quelques limitations dans la prévision des essais de gonflement hydraulique. 
L'objet de ce travail est d'intégrer un modèle de comportement élastoplastique anisotrope formulé en grandes déformations et couplé à l'endommagement ductile isotrope. Une théorie de plasticité non associée et non normale est utilisée avec deux contraintes équivalentes différentes intervenant dans la fonction de charge et dans le potentiel plastique afin d'améliorer la description de l'anisotropie (Runesson et al., 1989 ; Brunig et al., 1998 ; Stoughton, 2002 ; Badreddine, 2006). Un écrouissage mixte (cinématique et isotrope) non linéaire est pris en compte dans le modèle. La formulation est effectuée dans le cadre de la thermodynamique des processus irréversibles avec variables internes. Le couplage avec l'endommagement est effectué dans le cadre de la mécanique de l'endommagement continu avec la notion de variables effectives basées sur l'hypothèse d'équivalence en énergie totale (Saanouni et al., 1994). L'objectivité du modèle est assurée par l'utilisation du formalisme en référentiel tournant (Dogui, 1989; Sidoroff et al., 2001). La procédure d'intégration que nous avons adoptée repose sur la méthode de chargement incrémentale associée à la méthode de prédiction élastique - correction plastique proposée dans Simo et Ortiz, (1985). Un schéma de discrétisation purement implicite est utilisé pour discrétiser les relations d'évolution de l'ensemble des variables internes à l'exception des équations de l'écrouissage qui seront discrétisées par un schéma asymptotique (Freed et al., 1992 ; Chaboche et al., 1996 ; Hammi, 2000 ; Saanouni et al., 2001 ; Saanouni et al., 2003 ; Khelifa et al., 2004; Badreddine, 2006). La correction plastique se fait selon la normale à la surface du potentiel plastique sans normalité à la surface de charge. Par ailleurs, l'objectivité incrémentale est assurée par l'utilisation d'une cinématique incrémentale en référentiel tournant rigide (Hughes et al., 1980). En s'appuyant sur une procédure initialement proposée par (Simo et Taylor, 1985 ; Chaboche et al., 1996; Saanouni et al., 2001 ; 2003 ; Khelifa et al., 2004 ; Badreddine, 2006) les équations discrétisées à résoudre sont réduites à 8 équations scalaires au lieu de 21 équations. Ce système réduit et fortement non linéaire est résolu itérativement par un schéma de Newton.

Dans la première section nous exposons brièvement les équations constitutives du modèle couplé. Dans la deuxième section nous présentons les aspects numériques globaux et locaux d'implémentation dans le code de calcul ABAQUS/Explicit. La troisième et dernière partie est dédiée à l'application du modèle à la simulation de l'essai de gonflement hydraulique sur des tôles minces en acier inoxydable X6CrNi18-09 en utilisant des matrices circulaires et elliptiques.

\section{Equations constitutives du modèle}

Le modèle de comportement couplé à l'endommagement est formulé en grandes déformations plastiques avec l'hypothèse des petites déformations élastiques. Cette hypothèse permet d'aboutir à la décomposition additive du taux des déformations totales en partie élastique et partie plastique définie par la relation [2]. Par ailleurs, nous avons utilisé le formalisme en référentiel tournant pour assurer l'objectivité des équations constitutives (Dogui, 1989). L'orientation de ce dernier est définie, à tout 
instant, par le tenseur de rotation $\underline{Q}$ dont l'évolution est représentée par la relation [3] et dont l'orientation initiale est définie par $\underline{Q}_{0}$. Toute quantité tensorielle $\underline{T}$ définie par rapport à la configuration déformée est transportée dans la configuration initiale de référence par le biais de la rotation $Q$ :

$$
\bar{T}=\underline{Q}^{T} \underline{T} \underline{Q}
$$

où la variable barrée $\bar{T}$ est définie par rapport au référentiel tournant.

Les aspects théoriques liés à cette formulation peuvent être consultés dans (Saanouni et al., 2003; Badreddine et al., 2005; Badreddine, 2006). Nous nous contentons ici de résumer l'ensemble des équations du modèle dans le tableau 1.

\begin{tabular}{|c|c|}
\hline $\begin{array}{l}\text { Taux de déformations } \\
\underline{\bar{D}}=\underline{\dot{\bar{\varepsilon}}}_{e}+\underline{\bar{D}}_{p}\end{array}$ & [2] \\
\hline $\begin{array}{l}\text { Taux de rotations du référentiel tournant de Jaumann } \\
\underline{\dot{Q}} \underline{Q}^{T}=\underline{W} \text { avec } \underline{Q}(t=0)=\underline{Q}_{0}\end{array}$ & [3] \\
\hline Relations d'état & \\
\hline Elasticité: $\bar{\sigma}\left(\underline{\varepsilon}_{e}, d\right)=(1-d) \overline{\underline{\Lambda}}: \underline{\varepsilon}_{e}$ & [4] \\
\hline Ecrouissage cinématique: $\underline{\bar{X}}(\underline{\bar{\alpha}}, d)=\frac{2}{3}(1-d) C \underline{\bar{\alpha}}$ & [5] \\
\hline Ecrouissage isotrope: $\mathrm{R}(r, d)=(1-d) Q^{r}$ & [6] \\
\hline Endommagement: $Y\left(\underline{\varepsilon}_{e}, \underline{\alpha}, r, d\right)=\frac{1-}{2} \underline{\varepsilon}_{\iota}: \bar{\Lambda}:-\underline{\varepsilon}_{\epsilon}+\frac{1}{3} C \underline{\alpha}^{-} \underline{\alpha}_{\alpha}+\frac{1}{2} \mathcal{Q} r^{2}$ & [7] \\
\hline Potentiel plastique $\bar{F}$ et critère de charge $\bar{f}$ & \\
\hline $\begin{aligned} \bar{F}(\underline{\bar{\sigma}}, \bar{X}, R ; d)= & \frac{1}{\sqrt{1-d}} \bar{\sigma}_{p}(\bar{\sigma}-\bar{X})-\frac{R}{\sqrt{1-d}}+\frac{3 a}{4(1-d) C} \bar{X}: \bar{X}+\frac{b}{2(1-d) Q} R^{2} \\
& +\frac{s}{s+1}\left\langle\frac{Y-Y_{0}}{s}\right\rangle^{s+1} \frac{1}{(1-d)^{\beta}}\end{aligned}$ & [8] \\
\hline
\end{tabular}




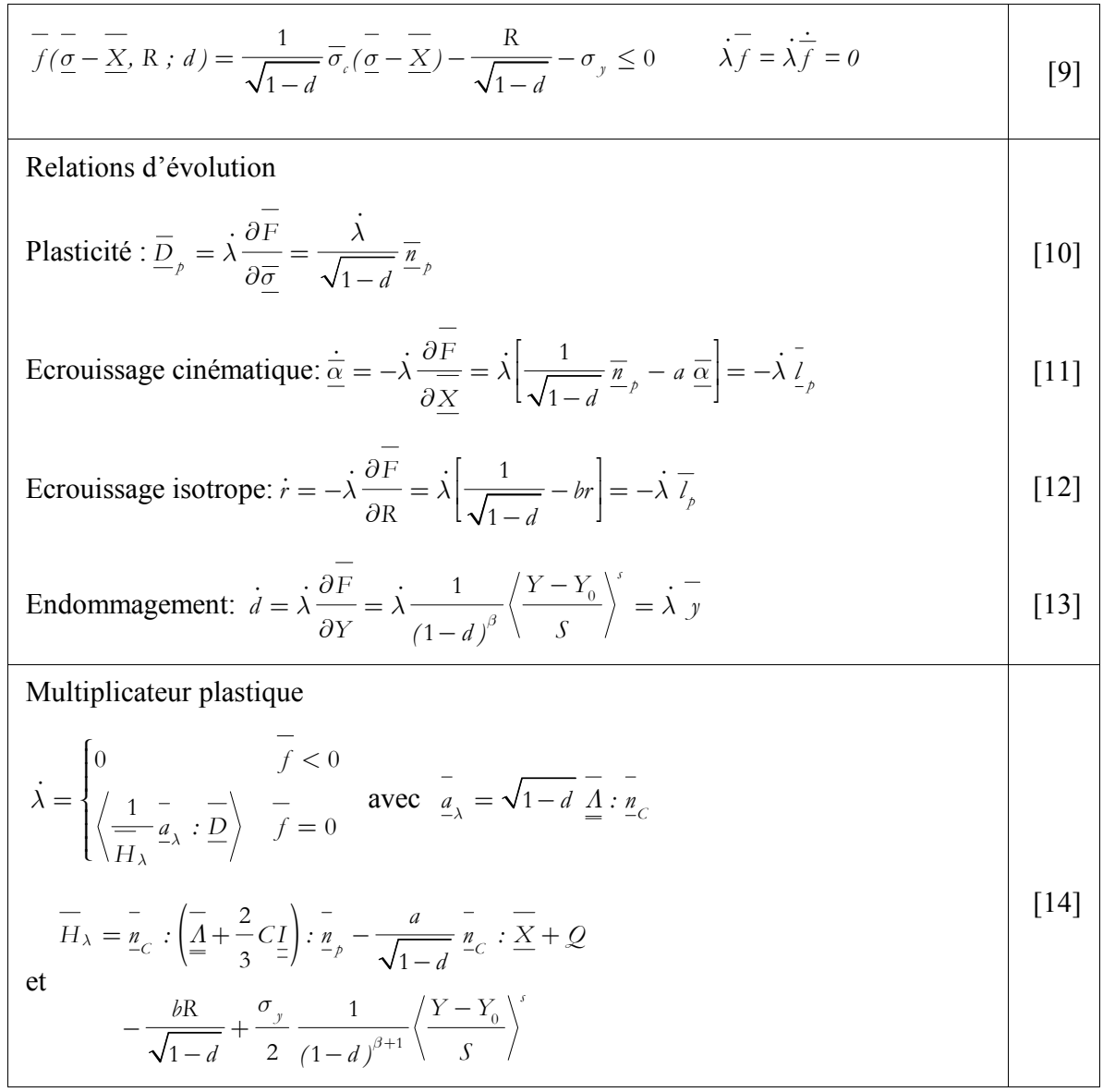

Tableau 1. Relations constitutives du modèle

$\underline{\underline{\Lambda}}$ est l'opérateur d'élasticité linéaire de l'élément de volume représentatif (EVR) fictif saint, dans le cas isotrope il est donné par $\underline{\underline{\Lambda}}=2 \mu_{l} \underline{\underline{I}}+\lambda_{l} \underline{1} \otimes \underline{1}$. C et $Q$ sont les modules de l'écrouissage cinématique et isotrope respectivement et les coefficients a et $\mathrm{b}$ caractérisent leur non-linéarité respective. $\sigma_{y}$ est la limite initiale d'élasticité, et $\beta, s, s$ et $Y_{0}$ sont des paramètres caractéristiques de l'évolution de l'endommagement ductile. La notation $\langle x\rangle$ symbolise la partie positive de $x$ définie par $\langle x\rangle=\max (x, 0)$. Les normes $\bar{\sigma}_{p}$ et $\bar{\sigma}_{C}$ sont des contraintes équivalentes, fonctions positives, convexes, homogènes de degré 1 , orthotropes de leurs arguments du type proposé par (Hill, 1948). Les formes explicites de ces contraintes sont les suivantes : 


$$
\bar{\sigma}_{p}^{2}=(\underline{\bar{\sigma}}-\overline{\bar{X}}): \underline{\underline{H}}^{\prime}:(\bar{\sigma}-\underline{\bar{X}}) \text { et } \bar{\sigma}_{C}^{2}=(\bar{\sigma}-\overline{\bar{X}}): \underline{\underline{H}}:(\bar{\sigma}-\overline{\bar{X}})
$$

où $\overline{\underline{H}}^{\prime}$ et $\underline{\underline{H}}$ sont les opérateurs d'anisotropie du potentiel plastique et du critère de plasticité respectivement. Ces opérateurs sont caractérisés respectivement par les constantes matérielles $\left(F^{\prime}, G^{\prime}, H^{\prime}, L^{\prime}, M^{\prime}\right.$ et $\left.N^{\prime}\right)$ et $(F, G, H, L, M$ et $N$ ). Ils ont une forme identique qui s'écrit pour l'opérateur $\underline{\underline{H}}$ sous la forme:

$$
\underline{\underline{H}}=\left[\begin{array}{cccccc}
G+H & -H & -G & 0 & 0 & 0 \\
-H & H+F & -F & 0 & 0 & 0 \\
-G & -F & F+G & 0 & 0 & 0 \\
0 & 0 & 0 & 2 N & 0 & 0 \\
0 & 0 & 0 & 0 & 2 M & 0 \\
0 & 0 & 0 & 0 & 0 & 2 L
\end{array}\right]
$$

Notons que le cas de la plasticité normale est obtenu en prenant $\bar{\sigma}_{C}=\bar{\sigma}_{p}$ ce qui revient à prendre $\underline{\underline{\bar{H}}}=\underline{\underline{H}}^{\prime}$.

Les tenseurs $\underline{\bar{n}}_{p}$ et $\overline{\bar{n}}_{C}$ donnés dans les relations [10] et [14] représentent respectivement la normale au potentiel et la normale au critère dans l'espace des contraintes. Compte tenu des formes des contraintes équivalentes données dans la relation [15], les normales ont les formes suivantes :

$$
\bar{n}_{p}=\frac{\underline{\underline{H}}^{\prime}:(\bar{\sigma}-\overline{\bar{X}})}{\bar{\sigma}_{p}} \quad \text { et } \quad \underline{n}_{C}=\frac{\underline{\underline{\bar{H}}}:(\overline{\bar{\sigma}}-\underline{\bar{X}})}{\bar{\sigma}_{C}}
$$

\section{Aspects numériques}

Le calcul des structures en mise en forme fait intervenir la résolution d'un problème d'équilibre non linéaire avec le comportement en grandes déformations élastoplastiques endommageables représenté par le modèle décrit précédemment et les relations de contact et frottement. Un tel problème ne peut être résolu que par des méthodes numériques notamment la méthode des éléments finis (MEF). Dans ce travail nous nous limitons à présenter la résolution du problème d'équilibre associé à un solide élastoplastique endommageable sans tenir compte des conditions de contact et frottement. Ces dernières, de type Coulomb, sont basées sur la notion de surfaces maîtres/esclaves disponible en standard dans (ABAQUS, 2004). 


\subsection{Formulation par éléments finis}

Tout problème d'équilibre peut être formulé en partant du principe des puissances virtuelles. Les formulations variationnelles en plasticité sont largement traitées dans des ouvrages de base (Bathe, 1981 ; Bonnet et al., 1997 ; Simo et al., 1998 ; Belytschko et al., 2000...). En recourant à une approximation nodale par sousdomaines (éléments finis) du champ des déplacements, la forme faible du principe des puissances virtuelles conduit au système algébrique suivant :

$$
[M]\{\ddot{u}\}+\left\{F_{\text {int }}\right\}-\left\{F_{\text {ext }}\right\}=0
$$

où la matrice masse globale et les vecteurs des efforts intérieurs et extérieurs sur toute la structure formée par $N_{e l}$ éléments de volume $V^{e}$, sont donnés par:

$$
[M]=\coprod_{e=1}^{N_{e l}}\left[M^{e}\right], \quad\left\{F_{i n t}\right\}=\coprod_{e=1}^{N_{e l}}\left\{F_{\text {int }}^{e}\right\}, \quad\left\{F_{e x t}\right\}=\coprod_{e=1}^{N_{e l}}\left\{F_{e x t}^{e}\right\}
$$

avec 【 est l'opérateur standard d'assemblage et :

$$
\begin{aligned}
& {\left[M^{(e)}\right]=\int_{V^{e}} \rho\left[N^{e}\right]^{T}\left[N^{e}\right] d V^{e}} \\
& \left\{F_{\text {int }}^{e}\right\}=\int_{V^{e}}\left[B^{e}\right]^{T}\left\{\sigma^{e}\right\} d V^{e} \\
& \left\{F_{\text {ext }}^{e}\right\}=\int_{V^{e}}\left[N^{e}\right]^{T}\left\{f_{v}^{e}\right\} d V^{e}+\int_{\Gamma^{e}}\left[N^{e}\right]^{T}\left\{f_{S}^{e}\right\} d S^{e}
\end{aligned}
$$

Les matrices $\left[N^{e}\right]$ et $\left[B^{e}\right]$ sont respectivement la matrice des fonctions d'interpolation et la matrice déformation-déplacement de l'élément solide.

La méthode la plus utilisée, en mise en forme pour résoudre le système [17], est la méthode incrémentale dynamique explicite (DE). L'objectif d'une telle méthode est l'obtention d'une solution en déplacement à la fin de l'incrément du temps $t_{n+1}=t_{n}+\Delta t_{n}$ en se basant exclusivement sur des quantités connues aux instants précédents. Cette méthode est utilisée dans le code ABAQUS/Explicit et est brièvement rappelée ici. 
En utilisant une matrice masse concentrée notée $\left[M_{C}\right]$ au lieu de la matrice masse consistante $[M]$ dans le système [17] écrit à l'instant $t_{n}$ la stratégie de résolution $\mathrm{DE}$ est donnée par :

1. résoudre le système [17] pour calculer les déplacements en fin de l'incrément du temps c'est-à-dire à $t_{n+1}$ :

$$
\begin{aligned}
& \{\ddot{u}\}_{n}=\left[M_{C}\right]_{n}^{-1}\left(\left\{F_{\text {int }}\right\}_{n}-\left\{F_{\text {ext }}\right\}_{n}\right) \\
& \{\dot{u}\}_{n+1 / 2}=\{\dot{u}\}_{n-1 / 2}+\frac{\Delta t_{n}+\Delta t_{n-1}}{2}\{\ddot{u}\}_{n} \\
& \{u\}_{n+1}=\{u\}_{n}+\Delta t_{n}\{\dot{u}\}_{n+1 / 2}
\end{aligned}
$$

2. intégrer les équations du comportement pour calculer la contrainte $\sigma_{n+1}$ et les variables internes à $t_{n+1}$ (Voir section 3.2);

3. calculer les quantités pour le pas suivant :

$$
\begin{aligned}
& {\left[M^{(e)} C\right]_{n+1}} \\
& \left\{F_{i n t}^{e}\right\}_{n+1} \text { par la relation [20] } \\
& \left\{F_{e x t}^{e}\right\}_{n+1} \text { par la relation [21] }
\end{aligned}
$$

le nouvel incrément de temps $\Delta t_{n+1}$ (voir ABAQUS, 2004)

4. si $t_{n+1}=t_{n}+\Delta t_{n+1}<\mathrm{T}(\mathrm{T}=$ temps total du chargement) reprendre l'étape (1), si non arrêt du calcul.

\subsection{Intégration locale : calcul des contraintes}

Pour calculer le vecteur des efforts intérieurs $\left\{F_{i n t}\right\}_{n+1}$ il faut calculer la contrainte en fin du pas de chargement. Ceci est réalisé en intégrant l'ensemble des relations constitutives du modèle discutées précédemment. Ces relations sont des équations différentielles ordinaires (EDO) définissant un problème d'évolution non linéaire. Ce problème d'évolution continue est transformé en un problème discret d'optimisation gouverné par le critère [9], grâce à l'approche de chargement incrémental utilisée avec la méthode de prédiction élastique - correction plastique due à (Simo et Ortiz, 1985). Cette approche consiste à appliquer à chaque incrément de temps un incrément de déformation $\Delta \underline{\varepsilon}$. Pendant cet incrément tous les champs 
$\left(\underline{\sigma}_{n}, \underline{\varepsilon}_{p n}, \underline{X}_{n}, \underline{Q}_{n}, R_{n}, d_{n}\right)$ sont supposés connus à l'instant $t_{n}$. Le problème est alors de déterminer ces champs en fin de l'incrément de temps que l'on note $\left(\underline{\sigma}_{n+1}, \underline{\varepsilon}_{p n+1}, \underline{X}_{n+1}, \underline{Q}_{n+1}, R_{n+1}, d_{n+1}\right)$ de sorte que le critère [9] soit identiquement vérifié. Une contrainte d'essai élastique notée $\underline{\sigma}_{n+1}^{t r}$ est calculée en supposant que l'incrément de déformation $\Delta \underline{\varepsilon}$ est purement élastique c'est-à-dire que les variables d'écrouissage et d'endommagement restent inchangées. Une correction plastique est opérée sur la contrainte et les autres variables de sorte que le critère plastique soit identiquement vérifié en fin du pas du temps.

Les relations d'évolution sont discrétisées en utilisant deux schémas d'intégration que sont la méthode d'Euler implicite et la méthode asymptotique (Freed et al., 1992). Cette dernière est utilisée essentiellement pour discrétiser les relations [15] et [16] d'évolution de l'écrouissage cinématique et de l'écrouissage isotrope.

Notons que pour assurer l'objectivité incrémentale, l'intégration du modèle s'effectue dans le référentiel tournant. C'est ainsi que les variables barrées sont définies par rapport à ce référentiel. La rotation du référentiel tournant est déterminée indépendamment des autres relations par la méthode de (Hughes et al., 1980) par :

$$
\underline{Q}_{n+1}=\left[\underline{1}-\frac{\Delta t}{2} \underline{W}_{n+\frac{1}{2}}\right]^{-1}\left[\underline{1}+\frac{\Delta t}{2} \underline{W}_{n+\frac{1}{2}}\right] \underline{Q}_{n}
$$

L'incrément de déformation $\Delta \underline{\varepsilon}$ est transporté par la rotation $\underline{Q}_{n+1}$ sur la base actuelle à $t_{n+1}$ du référentiel tournant :

$$
\Delta \bar{\varepsilon}=\underline{Q}_{n+1}^{T} \Delta \underline{\varepsilon}_{\underline{Q}+1}
$$

L'application du schéma d'Euler implicite aux équations [2] et [10] donne :

$$
\begin{aligned}
& \Delta \bar{\varepsilon}_{\underline{\varepsilon}}=\Delta \bar{\varepsilon}_{e}+\Delta \bar{\varepsilon}_{p} \\
& \Delta \underline{\bar{\varepsilon}}_{p}\left(\Delta \lambda, d_{n+1}, \overline{-}_{p n+1}\right)=\frac{\Delta \lambda}{\sqrt{1-d_{n+1}}} \bar{n}_{p n+1}
\end{aligned}
$$

La contrainte d'essai élastique est calculée en supposant que l'incrément de déformation $\Delta \underline{\varepsilon}$ est purement élastique : 


$$
\bar{\sigma}_{n+1}^{-t r}=\bar{\sigma}_{n}+\left(1-d_{n}\right) \underline{\Lambda}: \Delta \underline{\varepsilon}
$$

Compte tenu des équations [27], [28] et [29] la contrainte en fin de pas s'écrit :

$$
\bar{\sigma}_{n+1}\left(\Delta \lambda, d_{n+1}, \underline{-}_{p n+1}\right)=\frac{1-d_{n+1}}{1-d_{n}} \underline{-}_{n+1}^{-t r}-\Delta \lambda \sqrt{1-d_{n+1}} \underline{\underline{\Lambda}}: \underline{-}_{p n+1}
$$

Nous constatons que cette relation se compose d'une partie proportionnelle à la contrainte d'essai élastique et d'une partie proportionnelle à la normale au potentiel plastique en fin de pas. C'est ce terme qui pilotera la correction plastique.

En appliquant la méthode asymptotique aux relations d'évolution des écrouissages cinématique et isotrope, nous aboutissons aux contraintes d'écrouissage en fin de pas:

$$
\begin{aligned}
& \bar{X}_{n+1}\left(\Delta \lambda, d_{n+1}, \bar{n}_{p n+1}\right)=\frac{2 C\left(1-d_{n+1}\right)}{3}\left[e^{-a \Delta \lambda} \underline{\alpha}_{n}+\frac{1-e^{-a \Delta \lambda}}{a \sqrt{1-d_{n+1}}} \bar{n}_{p n+1}\right] \\
& \mathrm{R}_{n+1}\left(\Delta \lambda, d_{n+1}\right)=\mathcal{Q}\left(1-d_{n+1}\right)\left[e^{-b \Delta \lambda} r_{n}+\frac{1-e^{-b \Delta \lambda}}{b \sqrt{1-d_{n+1}}}\right]
\end{aligned}
$$

La discrétisation de la relation d'évolution [13] de l'endommagement par un schéma d'Euler implicite donne la variable d'endommagement en fin de pas :

$$
d_{n+1}\left(\Delta \lambda, d_{n+1}, \underline{-}_{p n+1}\right)=d_{n}+\frac{\Delta \lambda}{\left(1-d_{n+1}\right)^{\beta}}\left\langle\frac{Y_{n+1}\left(\Delta \lambda, d_{n+1}, \bar{n}_{p n+1}\right)-Y_{0}}{S}\right\rangle^{s}
$$

Toutes ces variables en fin du pas doivent vérifier le critère de charge :

$$
\bar{f}_{n+1}=\bar{\sigma}_{C}\left(\underline{\underline{n}}_{p n+1}, \Delta \lambda, d_{n+1}\right)-R_{n+1}\left(\Delta \lambda, d_{n+1}\right)-\sqrt{1-d_{n+1}} \sigma_{y}
$$

Les équations [28] et [30] à [34] forment un système non linéaire de 21 équations scalaires. Ce système d'équations non linéaires doit être résolu par un schéma itératif. Cette résolution est systématiquement réalisée en chaque point de Gauss de chaque élément et à chaque incrément de charge. Pour économiser le temps de calcul, il est avantageux de réduire ce système d'équations à seulement 8 équations, 
en exprimant toutes les variables en fonction de 3 variables principales que sont $\bar{n}_{p n+1}, \Delta \lambda$ et $d_{n+1}$. Ces 8 équations scalaires sont :

$$
\begin{aligned}
& \underline{-}_{n+1}\left(\underline{-}_{p n+1}, \Delta \lambda, d_{n+1}\right)=\overline{-}_{p n+1} \\
& -\frac{1}{\sigma_{p n+1}\left(\bar{n}_{p n+1}, \Delta \lambda, d_{n+1}\right)} \underline{\bar{H}}^{\prime}:\left[\begin{array}{l}
\bar{\sigma}_{n+1}\left(\underline{n}_{-p n+1}, \Delta \lambda, d_{n+1}\right) \\
-\bar{X}_{n+1}\left(\bar{n}_{p n+1}, \Delta \lambda, d_{n+1}\right)
\end{array}\right]=\underline{0} \\
& \bar{f}_{n+1}\left(\bar{n}_{p n+1}, \Delta \lambda, d_{n+1}\right)=\bar{\sigma}_{C}\left(\bar{n}_{p n+1}, \Delta \lambda, d_{n+1}\right) \\
& -R_{n+1}\left(\Delta \lambda, d_{n+1}\right)-\sqrt{1-d_{n+1}} \sigma_{y}=0 \\
& \bar{g}_{n+1}\left(\bar{n}_{p n+1}, \Delta \lambda, d_{n+1}\right)=d_{n+1}\left(\bar{n}_{p n+1}, \Delta \lambda, d_{n+1}\right)-d_{n} \\
& -\frac{\Delta \lambda}{\left(1-d_{n+1}\right)^{\beta}}\left\langle\frac{Y_{n+1}\left(\bar{n}_{p n+1}, \Delta \lambda, d_{n+1}\right)-Y_{0}}{s}\right\rangle^{s}=0
\end{aligned}
$$

La première équation n'est autre que l'équation de la normale au potentiel donnée par l'équation [16], la deuxième vient du critère d'écoulement donné par l'équation [34] et la troisième est donnée par l'équation [33] décrivant l'évolution de la variable d'endommagement. Ce système fortement non linéaire est résolu par la méthode itérative de Newton-Raphson. Dans le tableau 2 nous résumons les étapes de l'algorithme d'intégration adopté.

Cet algorithme est implémenté dans ABAQUS/Explicit via la routine utilisateur VUMAT. Il importe de signaler que si dans tous les points de Gauss d'un élément l'endommagement atteint la valeur critique $d_{C}=0.999$, cet élément est considéré totalement endommagé. Dans ce cas, toutes les contraintes sont alors nulles dans l'élément qui est éliminé par la procédure «Element Deletion » disponible dans ABAQUS en utilisant le critère $d \geq d_{C}$. La succession d'élimination des éléments totalement endommagés donne alors le chemin de la fissure macroscopique en propagation. 
1. Calcul de la rotation du référentiel tournant en fin du pas

$$
\underline{Q}_{n+1}=\left[1-\frac{\Delta t}{2} \underline{W}_{n+\frac{1}{2}}\right]^{-1}\left[1+\frac{\Delta t}{2} \underline{W}_{n+\frac{1}{2}}\right] \underline{Q}_{n}
$$

2. Transport rotationnel dans le référentiel tournant

$$
\Delta \underline{\varepsilon}=\underline{Q}_{n+1}^{T} \Delta \underline{\varepsilon} \underline{Q}_{n+1}, \quad \underline{\alpha}_{n}=\underline{Q}_{n}^{T} \underline{\alpha}_{n} \underline{Q}_{n}, \quad \bar{\sigma}_{n}=\underline{Q}_{n}^{T} \underline{\sigma}_{n} \underline{Q}_{n}, \quad \underline{X}_{n}=\underline{Q}_{n}^{T} \underline{X}_{n} \underline{Q}_{n}
$$

3. Prédiction élastique

$$
\overline{\bar{\sigma}}_{n+1}^{t r}=\underline{\bar{\sigma}}_{n}+\left(1-d_{n}\right) \underline{\bar{\Lambda}}: \Delta \underline{\bar{\varepsilon}}
$$

si $\bar{f}_{n+1}^{t r}\left(\underline{\sigma}_{n+1}^{-t r}-\bar{X}_{n}, \mathrm{R}_{n}, d_{n}\right)<0$ alors solution élastique :

4. $\quad \bar{\sigma}_{n+1}=\underline{\sigma}_{n+1}^{t r}, \quad \underline{\alpha}_{n+1}=\underline{\alpha}_{n}, \quad r_{n+1}=r_{n}, \quad d_{n+1}=d_{n}$

5. Transport rotationnel inverse dans la configuration actuelle.

$$
\underline{\alpha}_{n+1}=\underline{Q}_{n+1} \underline{\bar{\alpha}}_{n+1} \underline{Q}_{n+1}^{T}, \quad \underline{\sigma}_{n+1}=\underline{Q}_{n+1} \overline{\bar{\sigma}}_{n+1} \underline{Q}_{n+1}^{T}, \quad \underline{X}_{n+1}=\underline{Q}_{n+1}^{T} \bar{X}_{n+1} \underline{Q}_{n+1}
$$

Sortir et passer au pas suivant.

si $\bar{f}_{n+1}^{t r}\left(\underline{\sigma}_{n+1}^{t r}-\underline{X}_{n}, R_{n}, d_{n}\right) \geq 0$ alors correction plastique

4. Calcul des variables réduites $\underline{-}_{p n+1}, \Delta \lambda$ et $d_{n+1}$ par la méthode itérative de Newton-Raphson.

5. Calcul des variables internes auxiliaires

$$
\left(\underline{\bar{\sigma}}_{n+1}, \bar{\varepsilon}_{p n+1}\right),\left(\underline{X}_{n+1}, \bar{\alpha}_{n+1}\right),\left(R_{n+1}, r_{n+1}\right) \text { et }\left(Y_{n+1}, d_{n+1}\right) \text {. }
$$

6. transport rotationnel inverse dans la configuration actuelle.

$$
\underline{\alpha}_{n+1}=\underline{Q}_{n+1} \bar{\alpha}_{n+1} \underline{Q}_{n+1}^{T}, \quad \underline{\sigma}_{n+1}=\underline{Q}_{n+1} \bar{\sigma}_{n+1} \underline{Q}_{n+1}^{T}, \quad \underline{X}_{n+1}=\underline{Q}_{n+1} \bar{X}_{n+1} \underline{Q}_{n+1}^{T}
$$

Sortir et passer au pas suivant.

Tableau 2. Algorithme d'intégration 


\section{Application au gonflement hydraulique}

L'essai de gonflement hydraulique est un test qui permet de caractériser la formabilité des tôles minces en expansion sans influence importante du frottement. Pendant cet essai, un flan de forme circulaire de rayon $\mathrm{R}_{f}=66,5 \mathrm{~mm}$ et d'épaisseur $e_{f}=1 \mathrm{~mm}$ est bloqué entre une matrice et un serre-flan. Un jonc peut être utilisé pour empêcher la tôle de glisser radialement. Un fluide sous pression $P$ est alors injecté et provoque la déformation du flan par gonflement.

La matrice peut être de forme circulaire ou elliptique :

- la matrice circulaire est de diamètre extérieur $D_{m}=164 \mathrm{~mm}$ et de diamètre intérieur $d_{m}=91 \mathrm{~mm}$ avec un rayon de dégagement de $R_{m}=6 \mathrm{~mm}$ et une hauteur $H_{m}=25 \mathrm{~mm}$,

- la matrice elliptique est de grand axe $G_{A}=110 \mathrm{~mm}$, petit axe $P_{A}=74 \mathrm{~mm}$ avec un rayon de dégagement $R_{m}=6 \mathrm{~mm}$ et une hauteur de $H_{m}=25 \mathrm{~mm}$.

Les essais expérimentaux sont effectués au LGM-ENIM avec les deux types de matrice sur des flans en acier inox X6CrNi18-09. Les résultats sont exprimés en termes de pression appliquée en fonction du déplacement au pôle. Un palpage est effectué pour déterminer le profil du flan pour une pression donnée.

\subsection{Identification}

Pour identifier les paramètres des modèles avec plasticité normale et non normale donnés plus haut, nous disposons d'une base de données expérimentales fournie par le LGM-ENIM et qui comporte les courbes effort - déplacement obtenues suite à des essais de traction conduits jusqu'à rupture sur des éprouvettes de tôles minces en acier inoxydable X6CrNi18-09. Ces éprouvettes sont découpées selon un angle $\psi_{0}$ variant de $0^{\circ}$ à $90^{\circ}$ avec un pas de $15^{\circ}$ par rapport à la direction de laminage qui est prise dans la suite comme étant la direction 1. Les résultats expérimentaux comportent aussi la courbe d'évolution du coefficient de Lankford ( $\left.r={ }^{\varepsilon_{p 22}} / \varepsilon_{p 33}\right)$ en fonction de l'orientation $\psi_{0}$. Il importe de noter que l'évolution du coefficient de Lankford servira à identifier les paramètres d'anisotropie du potentiel plastique. Ces derniers seront déterminés de façon à approcher au mieux les courbes expérimentales sachant que trois directions suffisent pour déterminer les paramètres dans le plan de la tôle. Pour les paramètres d'anisotropie du critère de plasticité nous utiliserons uniquement les résultats expérimentaux relatifs aux orientations $0^{\circ}, 45^{\circ}$ et $90^{\circ}$.

Nous commençons dans une première étape par l'identification des paramètres matériau de chaque modèle en un point matériel en utilisant les courbes contrainte déformation issues des courbes expérimentales effort - déplacement avec une 
procédure d'optimisation. Dans une seconde étape nous utilisons l'ensemble des constantes matérielles déterminées dans l'étape précédente pour simuler numériquement l'essai de traction en respectant la géométrie de l'éprouvette utilisée. On apporte, le cas échéant, des corrections sur certaines constantes, généralement les constantes de dommage $S$ et $\beta$ afin de décrire au mieux la courbure de la réponse dans la phase adoucissante. Pendant cette étape on détermine la finesse optimale du maillage $\Delta x$ ainsi que le meilleur pas de chargement permettant de se rapprocher de la réponse expérimentale, essentiellement dans la phase d'adoucissement de la courbe exprimée en termes de force - déplacement.

Pour le modèle avec plasticité normale nous commençons par identifier la limite d'élasticité initiale $\sigma_{y}$ et les paramètres d'écrouissage $Q, b, C$ et $a$ avec un calcul non couplé effectué dans une fenêtre de déformation $\varepsilon<15 \%$, avec un modèle isotrope. Trois jeux de paramètres $\sigma_{y}, Q, b, C$ et $a$ sont obtenus relativement à chaque direction $\psi_{0}$. Une moyenne de ces paramètres est retenue et fixée ensuite pour identifier les paramètres d'anisotropie $F, G, H$ et $N$. Les paramètres $M$ et $L$ sont fixés à la valeur 1.5 qui correspond au cas isotrope. Les paramètres $Q, b, C, a, F, G, H$ et $N$ étant ainsi déterminés et fixés nous effectuons ensuite des calculs couplés pour déterminer les paramètres d'endommagement $s, S, \beta$ et $Y_{0}$.

Pour le modèle avec plasticité non normale nous déterminons les paramètres d'anisotropie du potentiel $F^{\prime}, G^{\prime}, H^{\prime}$ et $N^{\prime}$ qui permettent d'approcher au mieux la courbe d'évolution du coefficient de Lankford. Les autres paramètres sont déterminés par la même procédure expliquée pour le modèle avec plasticité normale.

Des simulations de l'essai de traction ont été effectuées avec deux maillages de finesse différente ( $\Delta x=1 \mathrm{~mm}$ et $\Delta x=0,5 \mathrm{~mm}$ ) avec des éléments hexaédriques à 8 nœuds et à intégration réduite (C3D8R). Ces deux maillages ont conduit à des solutions identiques jusqu'à la phase adoucissante à partir de laquelle une légère différence est observée. En revanche le temps de calcul induit par le maillage le plus fin $(\Delta x=0,5 \mathrm{~mm})$ est relativement plus important. C'est pour cette raison que nous avons retenu la taille d'élément $\Delta x=1 \mathrm{~mm}$ qui sera utilisée par la suite pour la simulation du gonflement hydraulique.

Les paramètres identifiés sont rassemblés dans le tableau 3. Ces paramètres ont permis d'avoir les courbes effort-déplacement données dans la figure 1 pour les 3 directions $0^{\circ}, 45^{\circ}$ et $90^{\circ}$. Ainsi que la courbe d'évolution du coefficient de Lankford donnée sur la figure 2. Notons que les courbes effort-déplacement sont pratiquement les mêmes pour les deux modèles (normal et non normal).

Dans toute la suite nous confrontons chaque fois qu'il est possible les réponses des modèles à plasticité normale et non normale avec les résultats expérimentaux chaque fois qu'ils sont disponibles.

Pour le modèle avec plasticité non normale, les résultats aussi bien en termes d'effort-déplacement (figure 1) qu'en termes d'évolution du coefficient de Lankford 
(figure 2) sont en bon accord avec l'expérience. Ce bon accord n'est pas réalisable avec un modèle de plasticité normale dans lequel la direction et l'intensité de l'écoulement sont gouvernées par la même fonction.

\begin{tabular}{|c|c|c|c|c|c|}
\hline Paramètres & $\sigma_{y}$ & E & $\nu$ & $Q$ & $b$ \\
\hline Unité & $\mathrm{MPa}$ & $\mathrm{GPa}$ & - & $\mathrm{MPa}$ & - \\
\hline Normal & 290 & 195 & 0.3 & 2682 & 1.65 \\
\hline Non normal & 290 & 195 & 0.3 & 2500 & 1.65 \\
\hline Paramètres & $C$ & $a$ & $S$ & $s$ & $\beta$ \\
\hline Unité & $\mathrm{MPa}$ & - & $\mathrm{MPa}$ & - & - \\
\hline Normal & 3650 & 75 & 600 & 1 & 4 \\
\hline Non normal & 3650 & 75 & 1000 & 1 & 8 \\
\hline Paramètres & $Y_{0}$ & $F^{\prime}$ & $G^{\prime}$ & $H^{\prime}$ & $L^{\prime}$ \\
\hline Unité & $\mathrm{MPa}$ & - & - & - & - \\
\hline Normal & 0 & 0.496 & 0.496 & 0.595 & 1.500 \\
\hline Non normal & 0 & 0.417 & 0.403 & 0.500 & 1.500 \\
\hline Paramètres & $M^{\prime}$ & $N^{\prime}$ & F & $G$ & $H$ \\
\hline Unité & - & - & - & - & - \\
\hline Normal & & 1.885 & 0.496 & 0.496 & 0.595 \\
\hline Non normal & 1.500 & 1.222 & 0.861 & 0.800 & 0.200 \\
\hline Paramètres & $L$ & $M$ & $N$ & $\rho$ & $\Delta x$ \\
\hline Unité & - & - & - & $\mathrm{g} \mathrm{mm}^{-3}$ & $\mathrm{~mm}$ \\
\hline Normal & 1.500 & 1.500 & 1.885 & $7.810^{-3}$ & 1 \\
\hline Non normal & 1.500 & 1.500 & 1.502 & $7.810^{-3}$ & 1 \\
\hline
\end{tabular}

Tableau 3. Ensemble des paramètres identifiés avec les modèles normal et non normal 
928 REMN - 16/2007. Modélisations numériques en mécanique

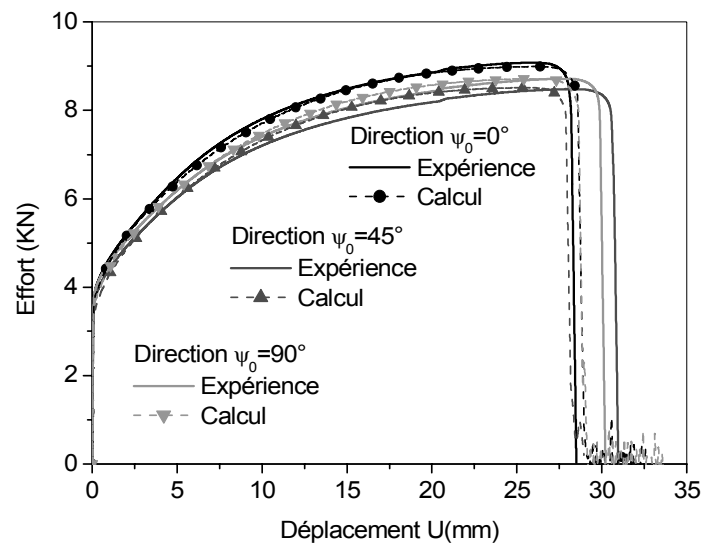

Figure 1. Courbes effort-déplacement résultats de l'identification obtenues avec les modèles normal et non normal pour les directions $0^{\circ}, 45^{\circ}$ et $90^{\circ}$

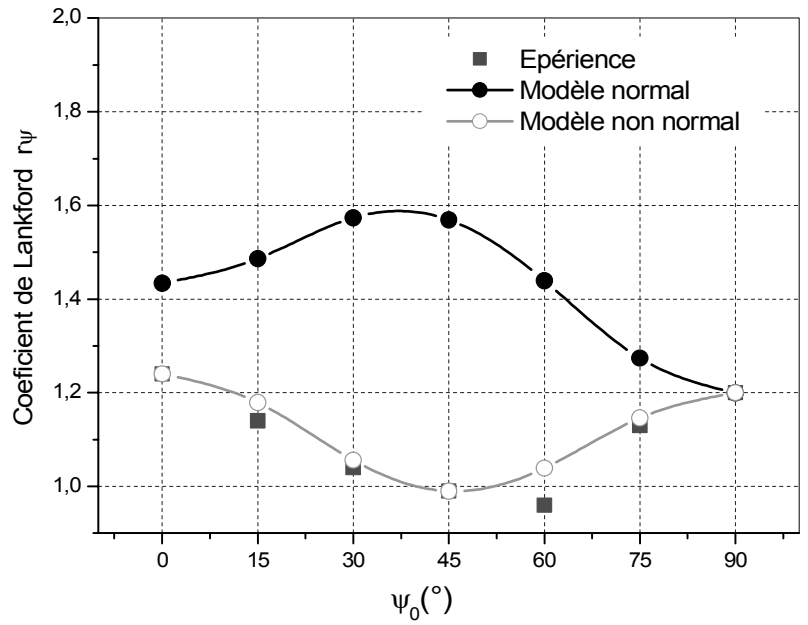

Figure 2. Evolution du coefficient de Lankford en fonction de l'orientation $\psi_{0}$ pour les modèles normal et non normal

\subsection{Résultats obtenus avec la matrice circulaire}

Les conditions de chargement ainsi que le maillage des composantes du procédé avec matrice circulaire sont présentés sur la figure 3 . 


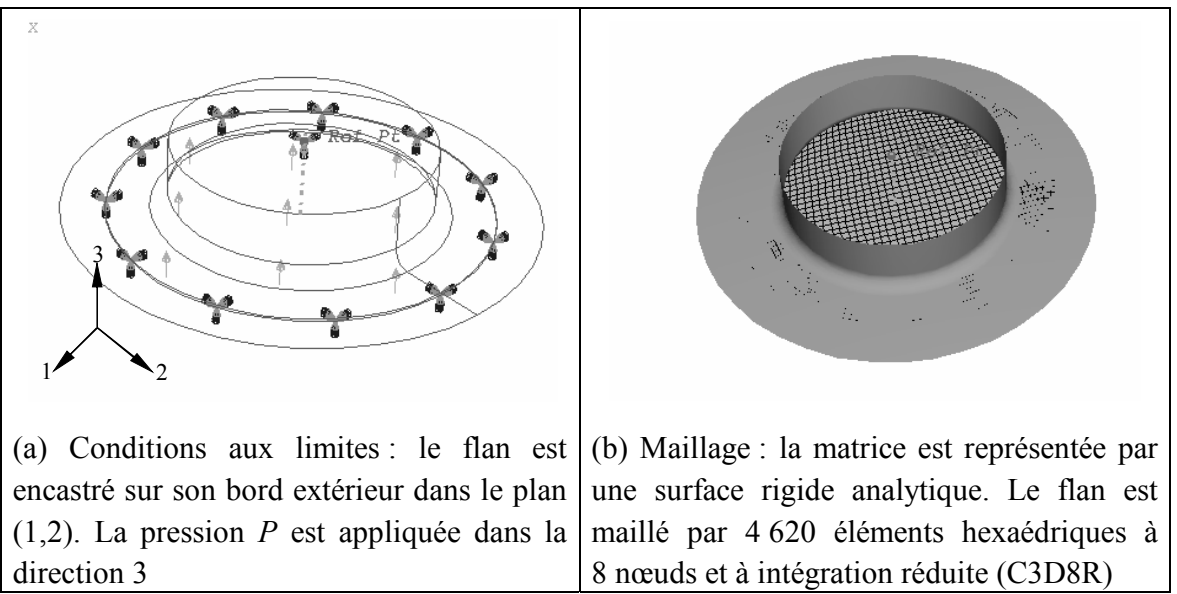

Figure 3. Conditions aux limites et maillage de l'essai avec matrice circulaire

Les isovaleurs en termes du second invariant de contrainte et d'endommagement obtenues avec le modèle non normal sont données dans la figure 4 pour deux pressions différentes (avant et après rupture). Nous constatons que pour la pression $P=235$ Bar (avant rupture) la distribution des champs est axisymétrique. Le champ de l'endommagement localise au niveau du pôle avec un fort gradient. La fissure s'amorce au pôle, conformément aux constations expérimentales, et se propage selon deux méridiennes en forme de croix.

La figure 5 montre une comparaison qualitative entre la fissure expérimentale et celle obtenue par simulation numérique. Dans les deux cas la fissure s'amorce au pôle et se propage le long d'une méridienne.

Sur la figure 6 sont reportés les résultats en termes de la pression appliquée en fonction du déplacement au pôle. Sur cette figure nous constatons que la solution numérique avec le modèle non normal est quasi confondue avec celle de l'expérience. Ceci n'est pas le cas avec la solution numérique délivrée par le modèle normal dont la surestimation de la pression augmente avec la croissance du déplacement au pôle. Il est à remarquer que la pression d'éclatement ( $P_{\mathrm{R}}=242 \mathrm{Bar}$ ) du flan est très bien prévue par le calcul du modèle non normal.

Les profils de la hauteur $\mathrm{z}$ en fonction de l'abscisse $\mathrm{x}$ selon une méridienne du flan gonflé à différentes pressions sont donnés sur la figure 7. En particulier pour la pression $P=176$ Bar, une comparaison avec le profil expérimental est donnée. Nous constatons clairement la supériorité du modèle non normal dans la prévision de la courbure du flan en forme de calotte sphérique. En effet le modèle normal sous estime significativement la hauteur du flan (figure 7). 
930 REMN - 16/2007. Modélisations numériques en mécanique

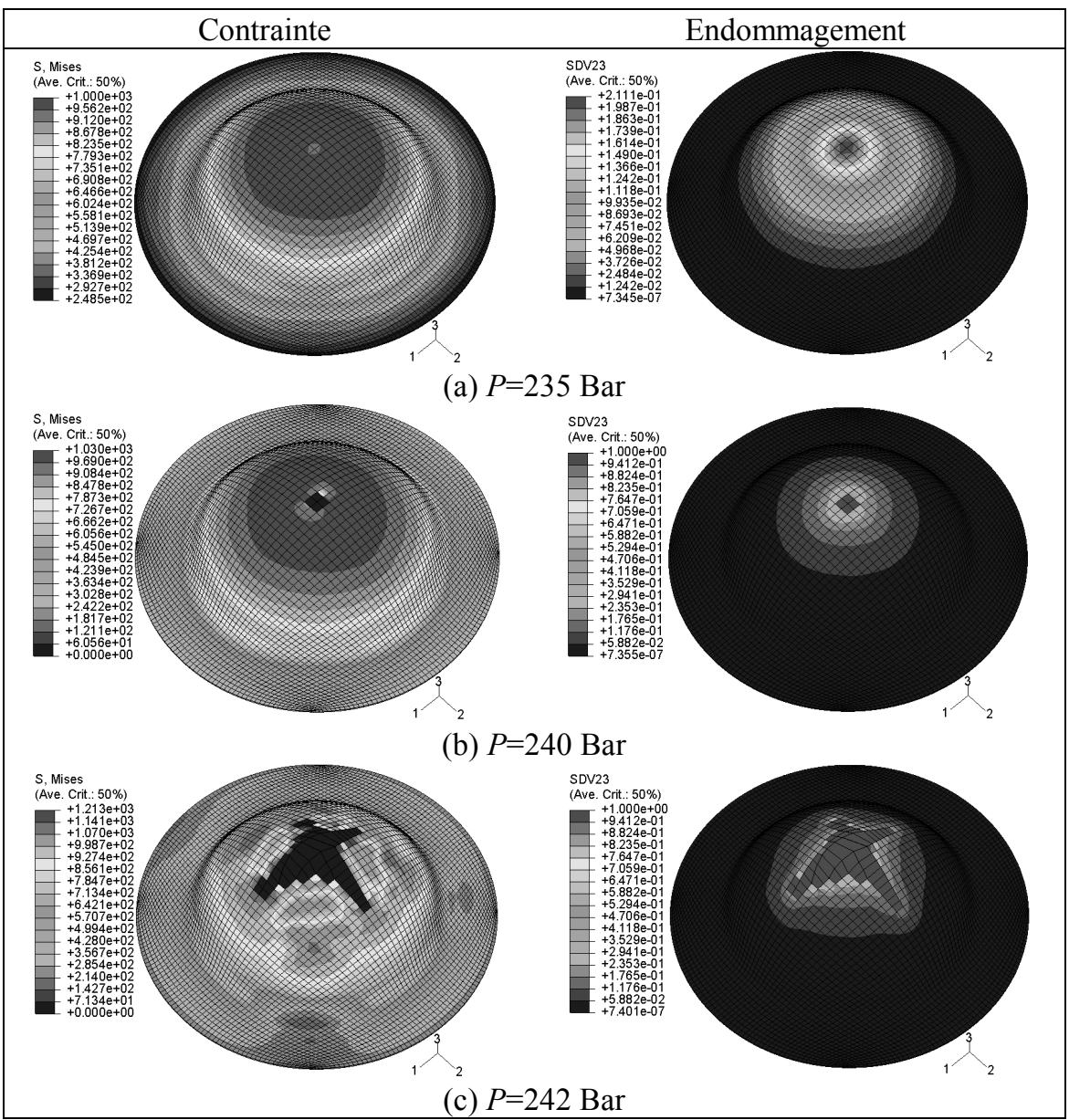

Figure 4. Isovaleurs obtenues avant et après rupture pour le cas de la matrice circulaire

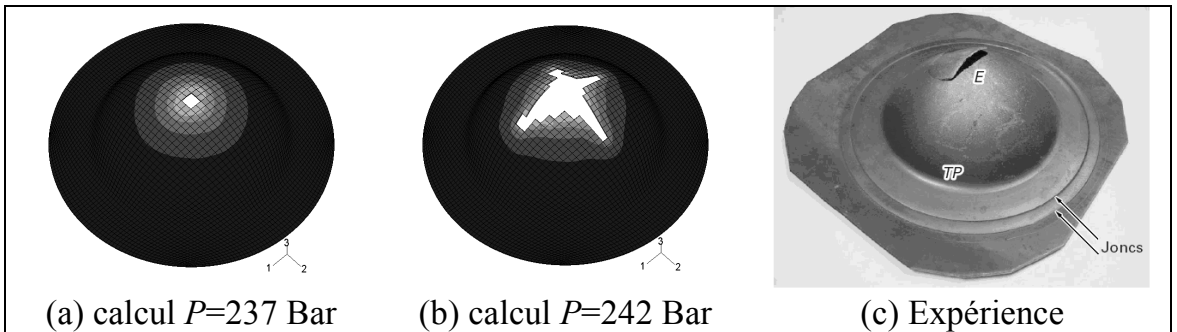

Figure 5. Comparaison calcul-expérience du profil de la rupture 


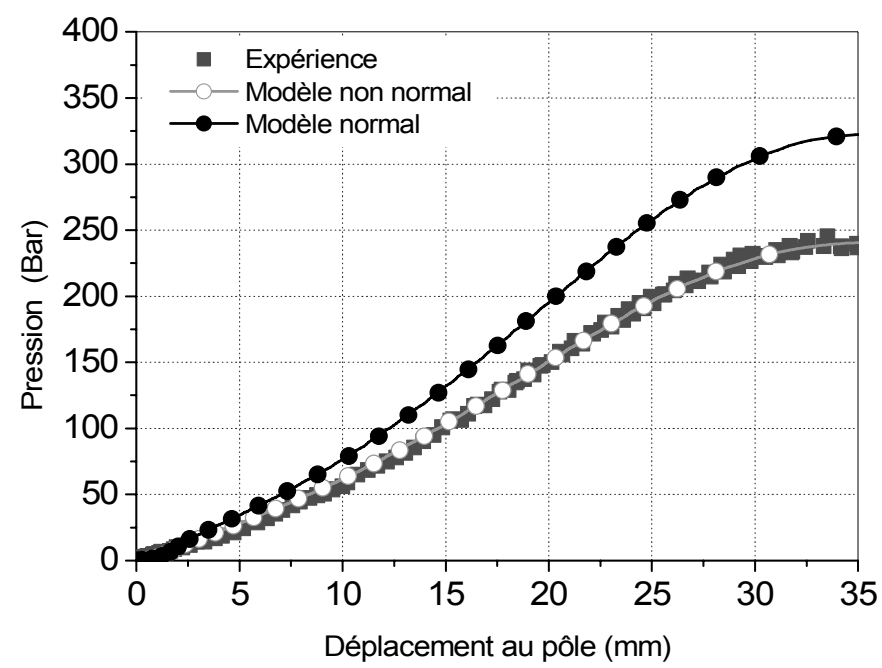

Figure 6. Comparaison calcul-expérience des courbes pression-déplacement

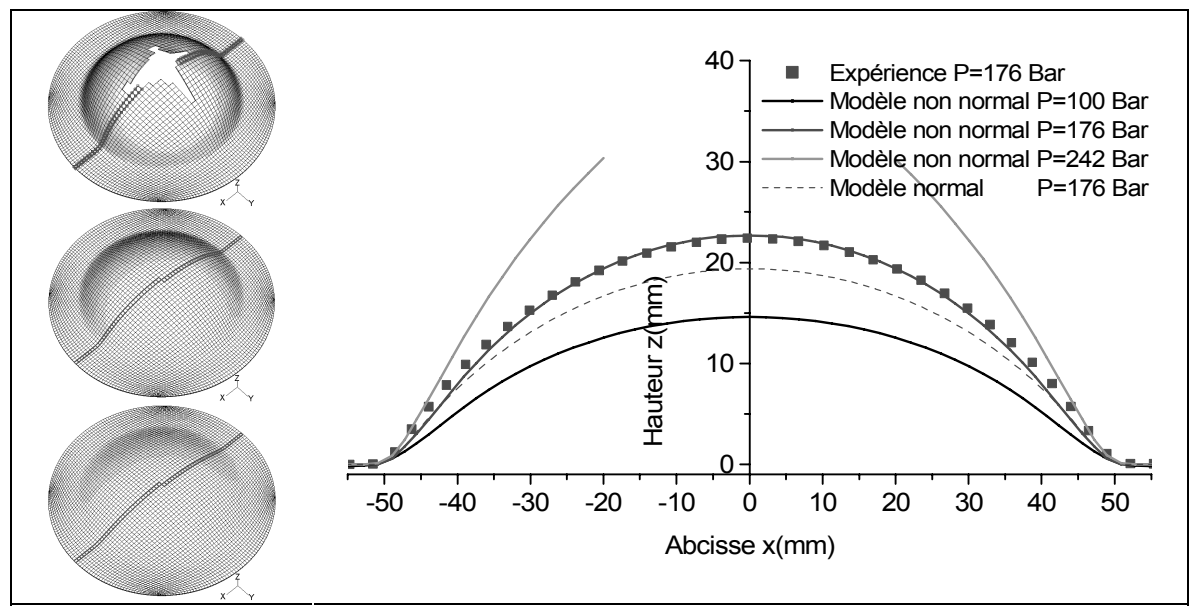

Figure 7. Comparaison calcul-expérience de la courbure du flan à différentes pressions

Sur la figure 8 nous reportons les trajets de déformation au pôle pour les deux modèles, exprimés en termes de déformation maximale $\varepsilon_{1}$ en fonction de la déformation minimale $\varepsilon_{2}$. Cette figure révèle que les trajets de déformation sont quasi confondus avec la droite d'équation $\varepsilon_{1}=\varepsilon_{2}$ qui correspond à l'expansion 
équibiaxiale. Notons que le modèle non normal conduit à un changement plus important avant rupture qui s'approche le plus de l'expansion équibiaxiale.

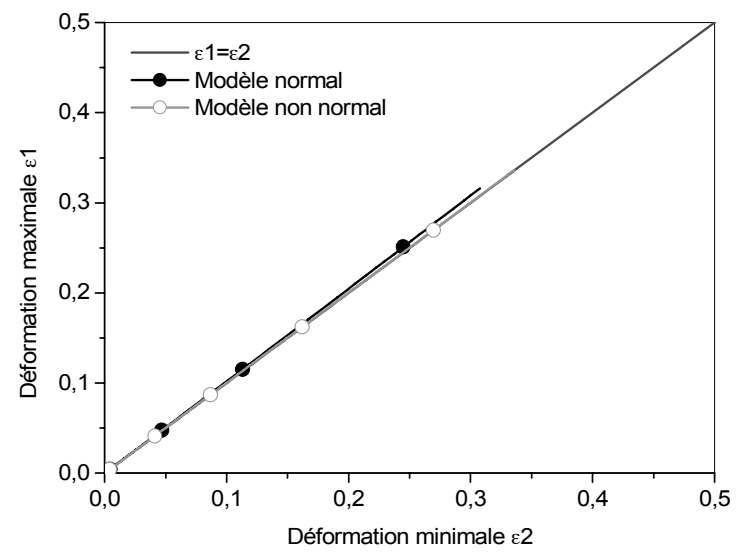

Figure 8. Trajets de déformation au pôle pour le cas de la matrice circulaire

\subsection{Résultats obtenus avec la matrice elliptique}

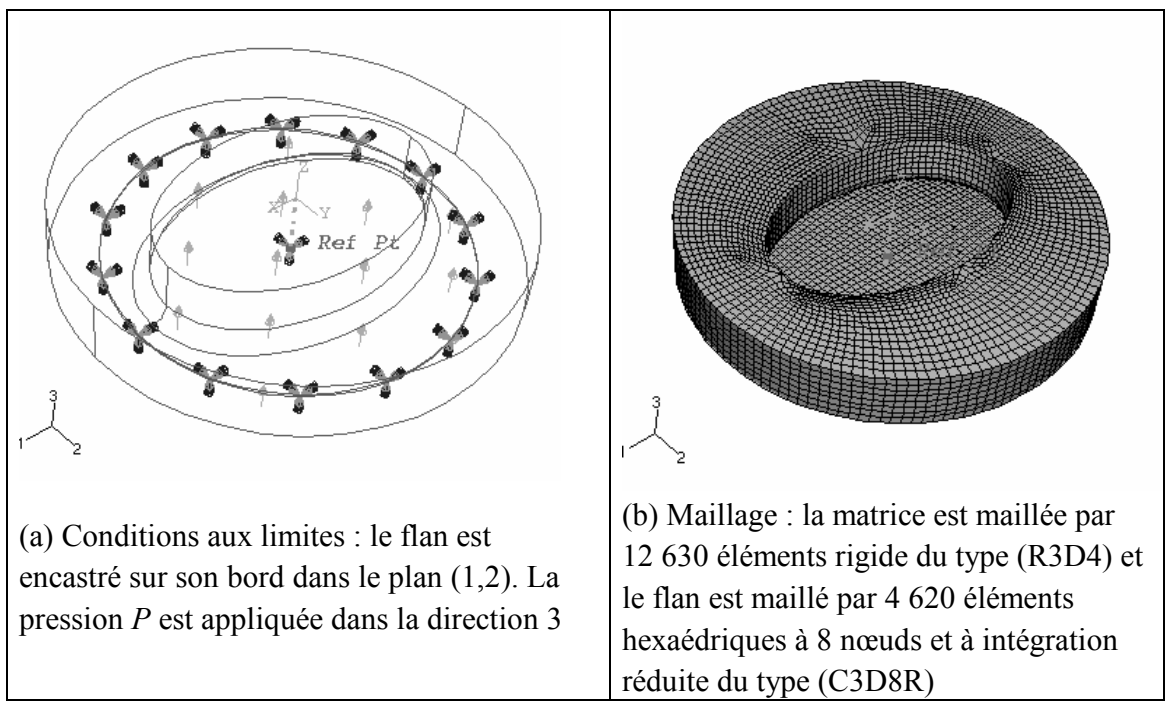

Figure 9. Conditions aux limites et maillage de l'essai avec matrice elliptique

Les conditions de chargement ainsi que le maillage des composantes de l'essai avec matrice elliptique sont présentés sur la figure 9 . 
De la même façon que pour l'essai avec la matrice circulaire, nous constatons d'après la figure 10 que le champ d'endommagement localise au pôle. La fissure s'amorce en ce point et se propage le long de la méridienne du grand axe de l'ellipsoïde. Notons que la distribution des champs ainsi que le trajet de la fissure ne sont pas influencés par l'orientation $\psi_{0}$ dans le plan $(1,2)$ du repère d'orthotropie.

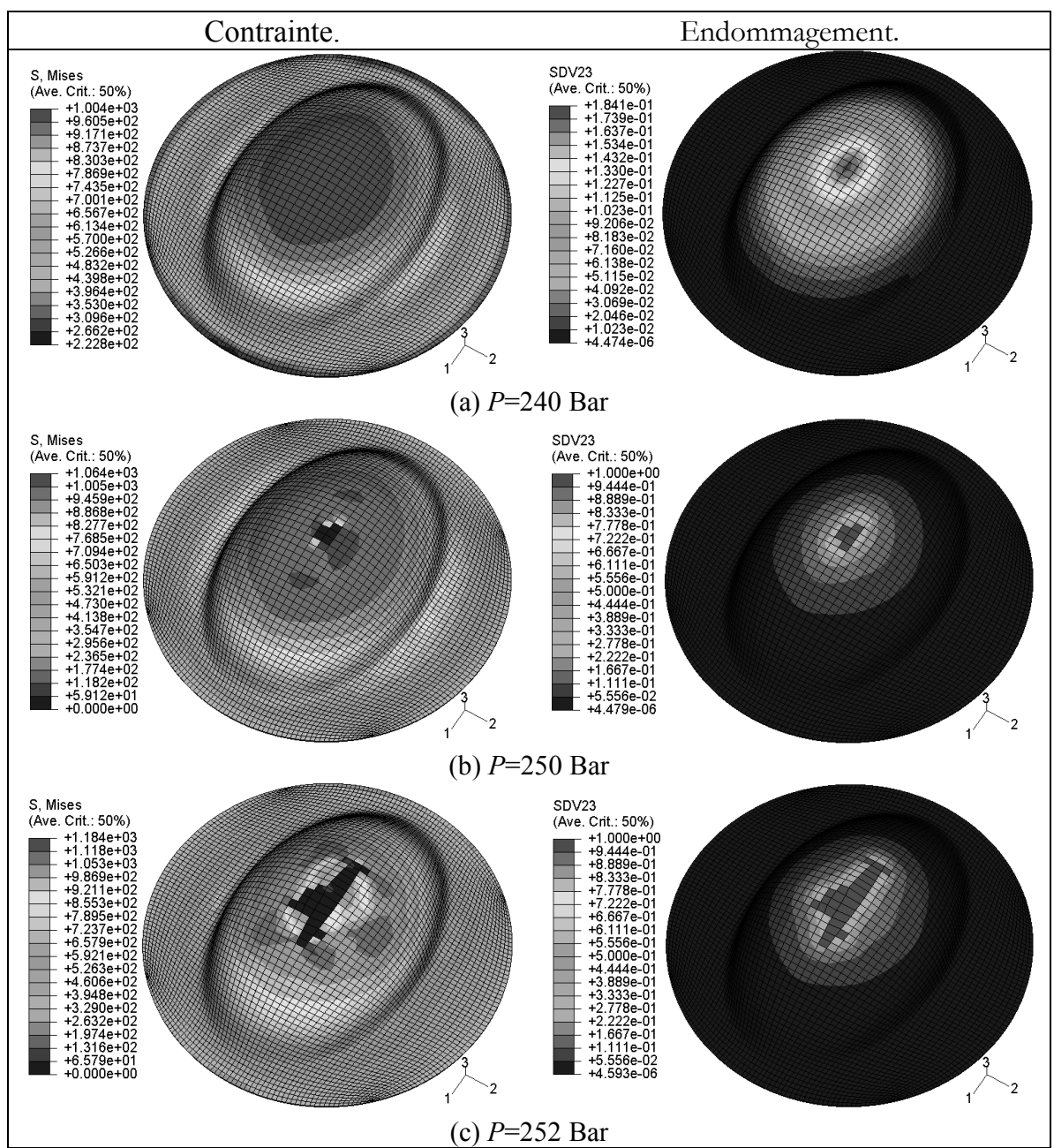

Figure 10. Isovaleurs obtenues avant et après rupture pour le cas de la matrice elliptique

Nous comparons sur la figure 11 la morphologie de la fissure obtenue expérimentalement avec celle obtenue numériquement par le modèle non normal. 
Nous remarquons que la fissure s'amorce au pôle et se propage selon le grand axe conformément à l'observation expérimentale.

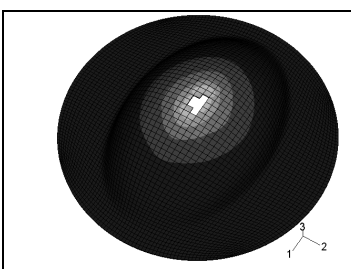

(a) calcul $P=250$ Bar

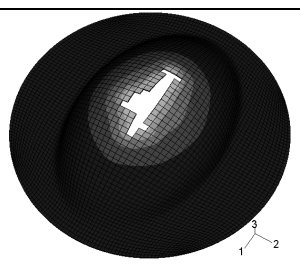

(b) calcul $P=252$ Bar

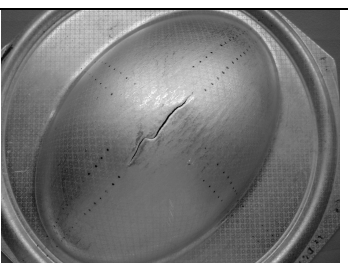

(c) Expérience

Figure 11. Comparaison calcul-expérience du profil de la rupture dans le cas de la matrice elliptique

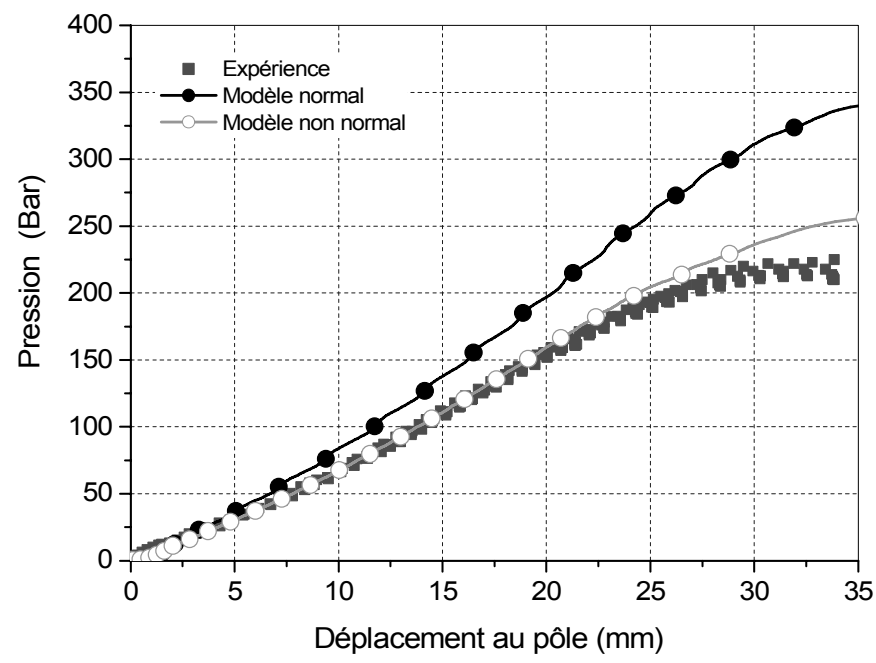

Figure 12. Comparaison calcul-expérience des courbes pression-déplacement pour le cas de la matrice elliptique

Les courbes pression-déplacement au pôle sont données dans la figure 12 pour la matrice elliptique. On note une bonne concordance entre les résultats issus du calcul avec le modèle non normal et l'expérience jusqu'à une pression $P=200$ Bar (qui correspond à un déplacement au pôle de l'ordre de $25 \mathrm{~mm}$ ). Au-delà de cette pression nous remarquons une légère différence avec la réponse expérimentale. Cette différence atteint son maximum à la rupture (ou l'éclatement) du flan. Le modèle non normal prévoit une explosion du flan à une pression $P=250$ Bar par contre l'explosion réelle s'effectue avec une pression un peu plus faible à peu prés $P=225$ Bar, soit une erreur relative de l'ordre de $11 \%$. En comparant ces résultats à 
ceux du modèle normal, nous remarquons encore une fois les meilleures performances prévisionnelles du modèle non normal. En effet, d'après la figure 12 la pression d'éclatement du flan prévue par le modèle normal est à peu prés de 340 Bar, ce qui fait une erreur de 51,1\% par rapport à l'expérience.

Sur la figure 13 nous reportons la hauteur $\mathrm{z}$ du profil du flan gonflé avec différentes pressions ( $P=100$ Bar, 160 Bar et 240 Bar) en fonction de l'abscisse $\mathrm{x}$ selon le grand et l'abscisse y selon le petit axe. Pour la pression $P=160$ Bar nous comparons avec un palpage réel du flan. Pour cette pression, la figure révèle que la simulation avec le modèle non normal donne une bonne prévision de la courbure du flan selon les deux axes ce qui n'est pas le cas avec le modèle à plasticité normale.

Les trajets de déformation au pôle pour les deux modèles, comme le montre la figure 14, diffèrent de l'expansion équibiaxiale. Ces trajets sont des droites de pente quasi identiques et à peu prés égales à 1.52. Cette valeur est à rapprocher du rapport entre le grand et le petit axe de l'ellipsoïde qui est de 1.48. On note qu'il s'agit d'un trajet d'expansion biaxiale qui se situe entre l'expansion équibiaxiale et la traction plane (droite $\varepsilon_{2}=0$ ).

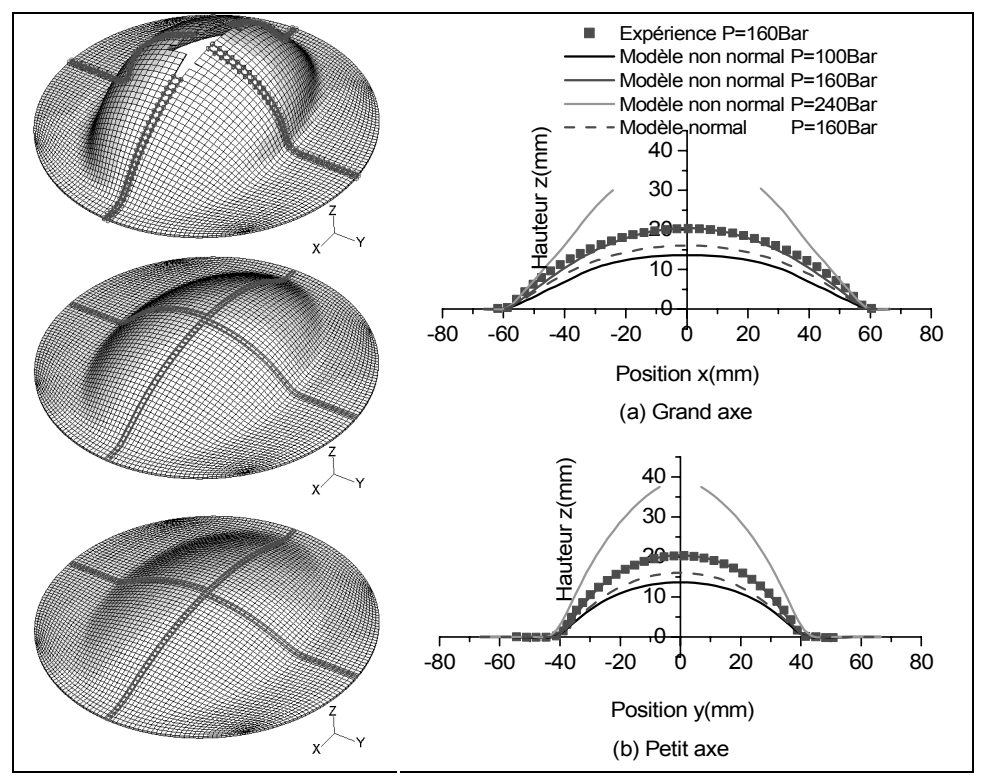

Figure 13. Comparaison calcul-expérience du profil du flan à différentes pressions (cas de la matrice elliptique) 


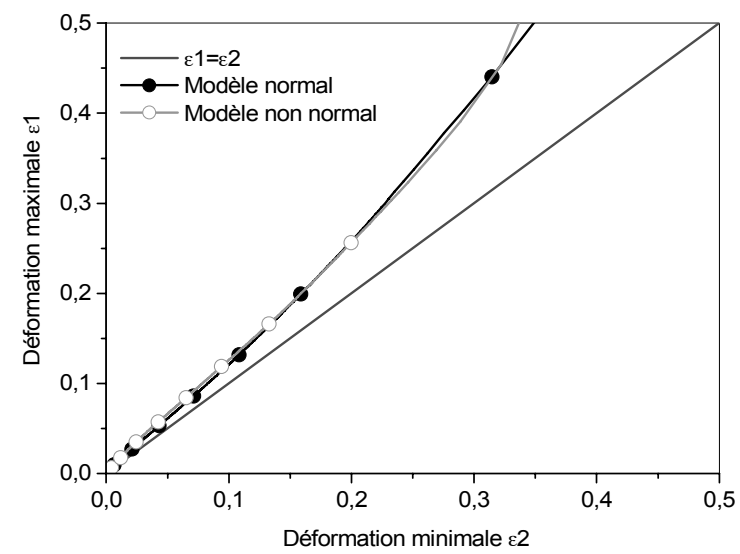

Figure 14. Trajets de déformation au pôle (cas de la matrice elliptique)

Pour terminer nous signalons que, s'agissant d'une modélisation locale avec adoucissement induit par le couplage comportement-endommagement, l'influence de la taille du maillage est importante. Nous avons en effet constaté que la finesse du maillage joue un rôle dans la phase post-critique (phase d'écrouissage négatif). Ce phénomène, hors du propos de cet article, ne peut être traité qu'avec des formulations non locales ou à gradient de variables d'état (Geers et al., 2003 ; Sornin et al., 2005, Sornin et al., 2006). Cependant, nous avons travaillé avec un maillage fixe dont la taille $\Delta x=1 \mathrm{~mm}$ à été fixée lors de l'identification des coefficients des modèles comme nous l'avons discuté dans la section 4.1. Il est avantageux d'utiliser une procédure de remaillage afin d'adapter la taille des éléments dans les zones où l'endommagement est actif. Une telle procédure a été appliquée en 2D (Borouchaki et al., 2002; Borouchaki et al., 2005; Cherouat et al., 2005). Sa généralisation au 3D est encore en cours de développement.

\section{Conclusions}

Un modèle de comportement élastoplastique anisotrope à plasticité non normale, couplé à l'endommagement ductile et formulé en transformations finies a été présenté ainsi que son implémentation dans le code de calcul ABAQUS/Explicit. Les résultats obtenus avec ce modèle sur des tôles en acier inoxydable X6CrNi18-09, aussi bien en traction uniaxiale qu'en expansion biaxiale (par gonflement hydraulique), montrent un très bon accord avec l'expérience. Une amélioration nette est effectivement remarquée par rapport aux résultats obtenus avec un modèle à plasticité normale. 
Des investigations sont en cours afin d'enrichir le modèle développé :

- par l'utilisation de critères de plasticité anisotrope non quadratiques afin de mieux traduire l'anisotropie des tôles pour divers matériaux,

- par une formulation non locale afin de s'affranchir de la dépendance vis-à-vis du maillage.

\section{Remerciements}

Les auteurs remercient pour leur soutien financier, le CRCA et le FEDER (contrat $\mathrm{n}^{\circ}$ 99-2-50-059) via le pole PMMC et le Comité Mixte de Coopération Universitaire Franco Tunisien (programme CMCU 01F1136).

\section{Bibliographie}

ABAQUS, Theory manual, Version 6.4, Hibbit, Karlson \& Sorensen, Inc, 2004.

Badreddine H., Saanouni K., Dogui A., «Nouvelle formulation en GD d'un modèle élastoplastique couplé à l'endommagement», CFM2005, $17^{e}$ Congrès Français de Mécanique, Troyes, 29 août, 2 Septembre, 2005.

Badreddine H., Elastoplasticité anisotrope en transformations finies: aspects théoriques, numériques et applications, Thèse de Doctorat, 2006, Université de Technologie de Troyes (France) - Ecole Nationale d'Ingénieurs de Monastir (Tunisie).

Bathe K.J., Finite element procedures in engineering analysis, Prentice Hall, 1981.

Belamri N., Cherouat A., Saanouni K., Autesserre P., «Simulation numérique du refendage de tôles minces ", CFM2005, $17^{e}$ Congrès Français de Mécanique, Troyes, 29 août, 2 Septembre, 2005.

Belytschko T., Liu W.K., Moran B., Nonlinear finite elements for continua and structures, John Wiley, New York, 2000.

Benallal A., Billardon R., Doghri I., "An integration algorithm and the corresponding consistent tangent operator for fully coupled elastoplastic and damage equations", Comm. Appl. Num. Methods, 4, 1988, p. 731-740.

Besson J., Cailletaud G., Chaboche J.-L., Forest S., Mécanique non linéaire des matériaux, Hermes Sciences Europe Ltd, Paris, 2001.

Bonnet J., Wood R.D., Nonlinear continuum mechanics for finite element analysis, Cambridge University Press, 1997.

Borouchaki H., Laug P., Cherouat A., Saanouni K., Adaptive remeshing for ductile fracture prediction in metal forming, C.R. Acd. Sci. Paris, Série. II b, Mécanique des solides et des structures, vol. 330, 10, 2002, p. 709-716.

Borouchaki H., Cherouat A., Laug P., Saanouni K., "Adaptive remeshing in large plastic strain with damage", Int. J. Numer. Meth. Engng, vol. 63, 2005, p. 1-36. 
Brunig M., Obrecht H., "Finite elastic-plastic deformation behavior of crystalline solids based on non-associated macroscopic flow rule", Int. J. Plasticity, 14, 1998, p. 1189-1208.

Cescotto S., Zhu Y.Y., "Non-linear dynamic analysis of 2D or 3D metal-forming processes by finite element", New advances in computational structural mechanics, P. Ladeveze and O.C. Zienkiewicz, Elsevier Science Publishers B.V., 1992.

Chaboche J.L., "Continuum damage mechanics, Parts I and II", J. of Ap. Mechanics, 55, 1988, p. 59-72.

Chaboche J.-L., Cailletaud G., "Integration methods for complex plastic constitutive equations", Computer methods in applied mechanics and engineering, vol. 133, Elsevier, 1996, p. 125-155.

Cherouat A., Borouchaki H., Saanouni K., Laug P., "Virtual metal forming with damage occurrence and adaptive remeshing", Int. J. of Forming Processes, vol. 8, $\mathrm{n}^{\circ} 2,2005$, p. 311-332.

Chiou Y.-J., Wang Y.-K., Hsiao P.-A. Chen, "Large displacement analysis of inelastic frame structures by convected material frame approach", Structural Engineering and Mechanics, vol. 13, $\mathrm{n}^{\circ}$ 2, 2002, p. 135-154.

Doghri I., "Fully implicit integration and consistent tangent modolus in elasto-plasticity", Int. J. Num. Meth; Engng., 1993, 36, p. 3915-3932.

Dogui A., Plasticité anisotrope en grandes déformations, Thèse de Doctorat d'Etat, Université Claude Bernard, Lyon, 1989.

Freed A. D., Walker K. P., "Exponential integration algorithm for first-order ODEs with application to viscoplasticity", ASME Summer Conf. On Mechanics and Materials Recent Advances on Damage Mechanics and Plasticity, Tempe, 1992.

Geers M., Ubachs R.L.J.M., Engelen R.A.B., "Strongly non-local gradient-enhanced finit strain elastoplasticity", Int Journal for Numerical Methods in Engineering, 56, 2003, p. 2039-2068.

Hambli H., "Finite element model fracture prediction during sheet-metal blanking processes", Eng. Fracture Mechanics, 2001, 68, p. 365-378.

Hammi Y., Simulation numérique de l'endommagement dans les procédés de mise en forme, PhD Thesis, University of Technology of Troyes, France, Avril 2000.

Hartley P., Clift S.E., Salimi J., Sturgess C.E.N., Pillinger I., "The Prediction of Ductile Fracture Initiation in Metalforming Using a Finite Element Method and Various Fracture Criteria”, Res. Mech., 1989, 28, p. 269-293.

Hill R., "A theory of yielding and plastic flow of anisotropic metals", Royal Soc, London Proc., 1948, p. 281.

Hughes T. J. R., Winget J., "Finite rotation effects in numerical integration of rate constitutive equations arising in large-deformation analysis", Int. J. Nume. Meth. Engng, 15, 1980, p. $1862-1867$.

Khan A.S., Huang S., Continuum theory of plasticity, John Wiley \&Sons, New York, 1995. 
Khelifa M., Badreddine H., Gahbiche M.-A., Saanouni K., Cherouat A., Dogui A., "Effect of anisotropic plastic flow on the ductile damage evolution in sheet metal forming, Application to the circular bulging test", International Journal of Forming Processes, vol. $8, \mathrm{n}^{\circ} 2,2005$, p. 271-289.

Khelifa M., Badreddine H., Saanouni K., Gahbiche M.-A., Dogui, A., «Plasticité anisotrope couplée à un endommagement ductile isotrope : appliction au gonflement hydraulique de tôles minces ", Revue européenne de mécaniques numériques, Numéro spécial issu du CFM'2005.

Lee H., Peng K.E., Wang J., “An anisotropic damage criterion for deformation instability and its application to forming limit analysis of metal plates", Eng. Frac. Mech., 1985, vol. 21, $\mathrm{n}^{\circ} 5$, p. 1031-1054.

Lemaitre J., Chaboche J.-L., Mécanique des milieux solides, Dunod, Paris, 1985.

Lemaitre J., A course on damage mechanics, Springer Verlag Editions, 1992.

Runesson K., Mrotz Z., “A note on non associeted plastic flow rules”, Int. J. Plasticity, 5, 1989 , p. 639.

Saanouni K., Forster C., Benhatira F., "On the anelastic flow with damage", Int. J. of Damage Mechanics, vol. 3, 1994, p. 141-169.

Saanouni K., Hammi Y., Numerical simulation of damage in metal forming processes, in Continuous Damage and Fracture, A. Benallal (ed.), Elsevier, 2000, p. 353-363.

Saanouni K., Cherouat A., Hammi Y., "Numerical aspects of finite elastoplasticity with damage for metal forming", European Journal of Finite Elements, vol. 10, n 2-3-4, 2001, p. 327-351.

Saanouni K., Chaboche J.L., "Computational damage mechanics, Application to metal forming", Chapter 7 of vol. 3, "Numerical and Computational methods", Ed. I. Miline, R.O. Ritchie and B. Karihaloo, Elsevier Oxford, 2003, p. 321-376.

Saanouni K., Mariage J.F., Cherouat A., Lestriez P., "Numerical prediction of discontinuous central bursting in axisymmetric forward extrusion by continuum damage mechanics", Computers \& Structures, vol. 82, 2004, p. 2309-2332.

Sidoroff F., Dogui A., "Some issues about anisotropic elastic-plastic models at finite strain", Int. J. Sol. Str., 38, 2001, p. 9569-9578.

Simo J. C., Taylor R. L., "Consistent tangent operators for rate-independent elastoplasticity”, Comput. Meth. Appl. Mech. Eng., vol. 48, 1985, p. 101-118.

Simo J.C., Ortiz M., "A Unified Approach to Finite Element Deformation Elastoplastic Analysis Based on the Use of Hyperelastic Equations", Comp. Meth. Appl. Meth. Engng., vol. 49, 1985, p. 221-245.

Simo J.C., Hughes T.J.R., Interdisciplinary Applied Mathematics, Mechanics and Materials, vol. 7, 1998, “Computational Inelasticity”, Springer - Verlag New York, Inc.

Sornin D., Saanouni K., "Theoretical and computational aspects of an elastoplastic dammge gradient non local model", Third European Conference on Computational Mechanics 
940 REMN - 16/2007. Modélisations numériques en mécanique

Solids, Structures and coupled Problems in Engineering, C.A. Mota Soares et al., (eds.), Lisbon, Portugal, 5-8 June 2006.

Sornin D., Saanouni K., "Formulation élastoplastique non locale en endommagement", CFM2005, $17^{e}$ Congrès Français de Mécanique, Troyes, 29 août, 2 Septembre, 2005.

Stoughton T. B., "A non associated flow rule for sheet metal forming”, Int. J. of plasticity, 18, 2002, p. 687-714.

Zhu Y.Y., Cescotto S., "The Finite Element Prediction of Ductile Fracture Initiation in Dynamic Metalforming Processes”, Journal de Physique III, 1, 1991, p. 751-757.

Zhu Y. Y., Cescotto S., Habraken A.M., “A fully coupled elastoplastic damage modeling and fracture criteria in metal forming processes", J. Mat. Proc. Tech., 1992, 32, p. 197-204. 